\title{
Theoretical trend of the covid19 spread from "birth to death" is a Gaussian within the Gaussian
}

\author{
Pardis Tabaee Damavandi
}

Subject to copyright law 2022. (C) All rights reserved.

The theoretical trend of the spread of the coronavirus and following mass vaccination is a "Gaussian within the Gaussian", with that I mean a Gaussian resulting from the sum of many Gaussians (waves), but the meaning of what the Gaussian entails is better understood in the case of the modern coronavirus, compared to the previous "Gaussian tendencies" that we observed in the smallpox and consequently smallpox vaccination case, in terms of the progression of the mutations, that will eventually lead to the "death of the coronavirus".

In truth, the above-mentioned trend exists in nature, as it happens in microbial competitions (virus against virus, and so on, which could be considered Lamarckian) and as everything has a beginning to an end, according to the prevalent thinking current, however, there is another novel Darwinian-inspired cogitation, to which the struggle to survive is what drives all organisms, which has been supported by misunderstood and documented Lazarus syndrome episodes. This could explain why suppressed viruses or bacteria existing time ago can always resurface if circumstances end up varying, however, there is no question that the vaccine is a deleterious circumstance for the virus, and that its role in terms of the "viral" trends to speed up the Gaussian throughout the period of time it weakens, and persists, until eradication.

Mass vaccination, it has been argued, must be carried out simultaneously, to support the best outcomes, rather than by a chronic management due to failed mass vaccinations, but the mRna virus was what became available in that time, and it had a narrow therapeutic index, whereby overdosages seemed to not have been ideal on the organism, and in that regard pharmacovigilance remains obviously always important.

Nevertheless, the eradication of the coronavirus will be a normal distribution, hence the previous articles I wrote, explained that the vaccine is going to be effective, but what emerges with the coronavirus pandemic is that the virus developed throughout the years, because of that Gaussian trend, where mutation over mutation morphed the virus to the stage in which it had an impact that suscitated a response or somewhat a "feedback" by the human organism, but what can be partially deduced and hypothesised by this and also through some bioinformatics tools is that the evolution of the virus is followed by a "devolution" of it too, 
by which the coronavirus, like other treated viruses, could get back to their primal state, unless there are other circumstances that can cause a diversion to the devolution, which are unfavoured in the context of mass vaccination. For instance, the latest omicron variant following vaccination, is a weakening to the virus that may be a regression to a "primitive" variant of the virus, and it wouldn't be too unusual that at some stage we get to a variant that may become "latent", notwithstanding exogenously, towards the human organism, and consequently common in other vectorial species; this is something that cannot be excluded, and probably likely, particularly because creating a new variant is less safe for a virus that must adapt to circumstances, and thus it makes sense that deviations to the devolution would likely occur in a setting that is coercitive, limitating to the virus, but an overpowering virus is unlikely with really good and qualitatively well-designed vaccines. One more reason why it would be less likely is that mass vaccination delivered in such a short period of time, is unlikely to provide differential circumstances, for that kind of deviation to occur, and in fact, through bioinformatics, we can observe some form of regression too, which is why, a simultaneous vaccination is best.

The concern that too many booster doses may cause less compliance is understandable, however, a simultaneous vaccination is unrealistic too, and ideally the response would be to make a potent vaccine, that lasts longer than a few months, and has a good therapeutic index.

\section{No conflicts of interest to disclose.}

\section{References}

1. Hornby K, Hornby L, Shemie SD (May 2010). "A systematic review of autoresuscitation after cardiac arrest". Crit. Care Med. 39 (5): 1246-53. 